\title{
An evidence-based practice project for recognition of clinical deterioration: Utilization of simulation-based education
}

\author{
Charyl Bell-Gordon, Elizabeth Gigliotti, Katy Mitchell \\ Nurse Practitioner Service, Houston Methodist Hospital, Houston, Texas, USA. \\ Correspondence: Charyl Bell-Gordon. Address: 6565 Fannin Street MGJ 11-002 Houston, Texas, USA. \\ Email: crbellgordon@houstonmethodist.org \\ Received: February 7, 2014 \\ Accepted: March 28, 2014 \\ Online Published: April 21, 2014 \\ DOI : 10.5430/jnep.v4n6p69 \\ URL: http://dx.doi.org/10.5430/jnep.v4n6p69
}

\begin{abstract}
Background: As more complex patients are hospitalized, the need for highly skilled and competent nurses to recognize clinical deterioration becomes more apparent. The literature supports the use of simulation-based education to enhance the recognition of clinical deterioration. The purpose of this evidence-based practice project was to utilize simulation as an educational modality to improve the knowledge of registered nurses in the recognition of clinical deterioration among their patients.
\end{abstract}

Methods: This evidence-based practice project was conducted from May through June 2013 in a 900-bed facility. Participation was voluntary and included 15 medical-surgical, procedural, and post-anesthesia care unit registered nurses. Simulation-based education was utilized for assessing the recognition, management, and reporting of clinical deterioration by nurses while supporting learning in a safe environment. Each participant managed two simulated patients in deteriorating states. Baseline performance was obtained during the initial simulation scenario by utilizing RAPIDS, a validated tool that evaluates assessment, management, and clinical deterioration reporting. A post-simulation debriefing and education session occurred that included a review of all required critical action elements. Debriefing was followed by a second post-intervention simulated clinical deterioration scenario.

Results: The results indicated statistically significant improvement in mean assessment and management scores when the post-intervention results were compared with baseline $[t(14)=2.04, p=.03]$. Post-intervention reporting scores were also improved, although this change was not statistically significant.

Conclusions: Simulation-based education may be an effective strategy for impacting a nurse's ability to recognize clinical deterioration and thereby allow for timely intervention.

\section{Key words}

Clinical deterioration, Simulation, Evidence-based practice, Registered nurse, RAPIDS

\section{I ntroduction}

The literature suggests that the clinical condition of patients admitted to medical-surgical units declines for several hours before recognition of this state. Failure to recognize clinical deterioration increases morbidity and mortality. As such, it is 
important for the nursing community to identify the reasons for this lack of recognition and to implement strategies to remedy this concern.

\subsection{Background}

As more complex patients are hospitalized, the need for highly skilled and competent nurses to recognize clinical deterioration becomes more apparent. Clinical deterioration is a concept frequently used in clinical practice yet is challenging to define. Triggers such as hypotension, hypertension, change in mental status, respiratory distress, decreased oxygen saturation, tachycardia, and bradycardia are sometimes used to describe indicators of clinical deterioration.

"There is increasing recognition that indicators of deterioration in acutely unwell adults are being missed" ${ }^{[1]}$ (p.724). Although abnormal vital signs are frequent occurrences in acute care units, there is minimal evidence of interventions that address these deteriorating states ${ }^{[2]}$. Clinical deterioration may go unrecognized secondary to nurses' lack of organization, knowledge, and supervision and failure to appreciate clinical urgency and seek advice ${ }^{[3]}$. Additional factors may include communication, staffing levels, task orientation, education, and teamwork. Nurses have the opportunity to improve patient safety by making it their highest priority ${ }^{[4]}$.

The complexity of medical-surgical patients is increasing, which places them at higher risk for deterioration. Steen ${ }^{[5]}$ reports that delayed recognition of clinical deterioration is suboptimal care, results in inappropriate admissions to the intensive care unit (ICU), and increases morbidity and mortality. The mortality of patients transferred from medicalsurgical acute care units to the ICU is significantly higher than that of patients admitted from the emergency department or operating rooms. Eighty-five percent of all in-hospital cardiac arrests are preceded by clear signs of deterioration that are often undetected ${ }^{[5]}$. Early recognition and timely interventions for clinical deterioration on acute care units may preclude ICU transfers and increased morbidity and mortality. Targeted strategies to identify clinical deterioration can improve early recognition.

In addition to the avoidable morbidity and mortality, unrecognized deterioration requiring transfer to a higher level of care such as an ICU creates a significant financial burden on the patient, hospital, insurance carriers, and tax payers. ICUs are one of the most expensive areas in a hospital. "In 2000, ICU costs exceeded \$55 billion, accounting for 13.3\% of hospitalization costs and $4.2 \%$ of national health care expenditures. Intensive care unit bed costs per day typically exceed

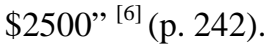

\subsection{Literature review}

Concept-associated terms are knowledge, recognition, and clinical deterioration. Chinn and Kramer ${ }^{[7]}$ describe knowledge as "knowing that is expressed in a form that can be shared or communicated with others" (p. 3). Knowing is an abstract concept that involves perceptions, experiences, and unconscious thoughts. A nurse combines all of the sources of knowing to articulate nursing knowledge of a subject such as clinical deterioration. Recognition can be described as perception, knowing, and/or identification.

Clinical deterioration is a concept that is relatively common to most clinicians. Although common, the clinical picture that one envisions may vary on the basis of education, specialty, and experience. Clinical is defined as treatment of patients ${ }^{[8]}$ (p. 134). The definition of deterioration is to make or become bad or worse ${ }^{[8]}$ (p. 201). Therefore, clinical deterioration in an acute adult medical-surgical setting is the worsening of a patient's condition from baseline requiring medical intervention.

Steen ${ }^{[5]}$ suggests that early recognition of and prompt intervention for clinical deterioration decreases ICU admissions and reduces in-hospital cardiac arrests. Steen also suggests that utilizing a systematic approach to patient assessment reduces the possibility of omission of critical steps. According to Steen ${ }^{[5]}$, the ABCDE (airway, breathing, circulation, disability, 
exposure) mnemonic is a systematic approach to the assessment of acutely ill patients that includes the look, listen, and feel that describe the appropriate interventions to be initiated when areas of concern are identified.

Endacott et al. ${ }^{[2]}$ embraced the importance of trends in identifying deterioration. Their study also identified the need for a systematic assessment because patient deterioration is often an anxiety-producing situation for the nurse. Endacott et al. ${ }^{\text {[2] }}$ recognized simulation as a useful tool to allow students to become familiar with the recognition of deterioration.

Anxiety or inexperience may impact a nurse's performance during an emergent clinical situation. Recognizing this possibility, both sources of evidence support a systemic approach to patient assessment. A systematic approach creates an organized structure that limits the possibility of overlooking a critical element.

Liaw and colleagues ${ }^{[9]}$ report knowledge and experience as important factors affecting a nurse's ability to recognize cues of patient deterioration. Additionally, the ability to synthesize collected cues and link them to the clinical presentation is equally important. Ineffective communication between nursing and medical staff was also identified when seeking help for a deteriorating patient. Liaw et al. ${ }^{[9]}$ also categorize the educational needs of the nurses into three broad areas: recognition, notification, and response. Buykx et al. ${ }^{[10]}$ concur that failure to recognize patient deterioration is a significant problem that can be improved with targeted education.

Educational programs including simulation can increase nursing competency in assessing and managing patient deterioration. One such program is FIRST2ACT (feedback incorporating review and simulation techniques to act on clinical trends). This is a simulation-based educational model that incorporates developing core knowledge, assessment (learning stimulus), simulation, reflective review, and performance feedback in order to assist nurses in their ability to assess and respond to clinical deterioration ${ }^{[10]}$. Evaluation of the program has suggested significant improvement in self-rated knowledge, self-rated confidence, and competence.

To evaluate the learning outcomes of simulation, Liaw and colleagues ${ }^{[11]}$ conducted "a prospective, randomized controlled trial with a pretest-posttest design” (p. 1225). The study was conducted with 31 participants. Participants reported positive simulation experiences with increased self-confidence ${ }^{[11]}$. Students who received the training had significantly higher posttest scores than did the control group.

Adequate evidence supports education as a strategy to increase nursing knowledge of clinical deterioration. The literature supports evaluating the nursing knowledge of deterioration in a simulated environment. In both cases, post-intervention results were more favorable than results before the intervention.

Liaw and colleagues ${ }^{[12]}$ describe the process of developing and validating the Rescuing a Patient in Deteriorating Situations (RAPIDS)-Tool. Based on literature review and national experts' consensus, the RAPIDS-Tool outlines the critical action elements for assessment and reporting of clinical deterioration. The RAPIDS-Tool divides the assessment items into the five categories of Airway, Breathing, Circulation, Disability, and Expose/Examine (ABCDE) and the four reporting categories of Situation, Background, Assessment, and Recommendation (SBAR). The validity of the tool was assessed by an international panel including physicians and critical care nurses. The validation process also included psychometric and construct validity testing. Significant differences $(t=15.48, p<.001)$ were appreciated in the performance scores of participants with different levels of training. According to Liaw et al. ${ }^{[12]}$, the RAPIDS-Tool demonstrated a high inter-rater reliability (ICC $=0.99$ ) among three raters and a high correlation between the global rating and checklist score $(r=0.94, p<.001)$. The RAPIDS-Tool was found to be reliable and valid for the evaluation of clinical deterioration in a simulated setting.

The previously described and validated RAPIDS-Tool was used by Liaw et al. ${ }^{[11]}$ to evaluate the performance of nursing students in the presence of clinical deterioration scenarios. A mean difference in total ABCDE score between baseline and after the educational program of 9.76 with a p-value at the significance level of 0.001 was reported. That study also 
revealed a difference in mean SBAR score of 3.30 at the significance level of 0.01 . Liaw et al. ${ }^{[11]}$ concluded that assessment, management, and reporting competencies in nursing students can be enhanced through a simulation-based educational program that includes the use of mnemonics.

\subsection{Purpose}

This evidence-based practice project was designed to enhance the staff nurse's ability to recognize acute changes in a patient's condition that warrant intervention. The project utilized simulation as a modality for assessment and providing education in a safe environment. The project focused on identifying the immediate actions that must be taken when acute changes occur as well as on reporting relevant clinical findings to a provider for supplementary clinical guidance.

\section{Methods}

The practice change project was implemented in a 900-bed Magnet-designated hospital in the Texas Medical Center. Prior to implementation, the project received approval from the Chief Nurse Executive and the Institutional Review Board.

Participation was voluntary, and focus areas included acute care units, high-risk procedural areas, and post-anesthesia care units (PACUs). Critical care nurses were excluded from the participant group because of enhanced nurse-to-patient ratios and access to advanced monitoring. All nurses working within the designated units were invited to participant. Age, gender, race, ethnicity, and years of nursing experience were not factors that were used to exclude participants. In fact, age, race, and ethnicity were not elements captured for the demographic evaluation.

Volunteers for the project were elicited through the use of a flyer that was emailed and placed on targeted units. Simulation sessions were scheduled for 3.5 hours over a 5-week period. A maximum of two participants were scheduled per session.

Confidentiality of participant results was maintained through random selection of a unique number. The number was used as the participant identifier. The same identifier was used for the initial and subsequent simulation sessions.

Before conducting the simulation sessions, the project manager, a nurse practitioner from the rapid response team, and a registered nurse with acute care experience evaluated each element of the validated RAPIDS-Tool for applicability to the scenarios used. To ensure that the total ABCDE score for each scenario in this project was equal to the ABCDE score described in the Liaw et al. ${ }^{[11]}$ benchmark study, 26 of the 31 elements were used for each scenario. However, the 26 elements may have differed by scenario on the basis of applicability. Elements that were not applicable to the scenario were removed, thereby generating an overall potential score of 36 rather than 41. Although the potential maximum ABCDE score for the RAPIDS-Tool was 41, the maximum ABCDE score for this project was 36 in alignment with the benchmark study. All of the SBAR components were used as in the benchmark study. The maximum total SBAR score was 20 .

Each volunteer participated in two different simulation scenarios with patients in deteriorating states. Baseline performance was obtained during the first simulation scenario. Performance of the nurse was assessed by utilizing the RAPIDS-Tool.

A post-simulation debriefing and education session lasting 1 hour occurred that included review of all required critical action elements described in the RAPIDS-Tool. Debriefing engages the participant and clarifies thought processes. According to Mayville ${ }^{[13]}$, “debriefing is reflective practice at its best and a critical element in the learning process” (p. 35). With the additional knowledge acquired during the debriefing, the participant underwent a second clinical deterioration simulation scenario followed by a second shorter debriefing. 
Performance review evaluated the participant's ability to systematically assess and report clinical findings. Patient assessment and management were evaluated with the ABCDE mnemonic. The nurse's reporting skills were assessed with the SBAR mnemonic. Each essential element on the mnemonic has several critical action components that generate a yes or no response. The sum of the total ABCDE and total SBAR scores generated the total RAPIDS performance score.

\section{Results}

Fifteen volunteers participated in the evidence-based practice project. Fourteen (93\%) were female and one (7\%) was male. Years of registered nurse experience ranged from 1 to 30 years. Years of tenure at the project implementation site ranged from 1 to 26 years (mean, 10.66 years). Fourteen of the participants (93\%) held a bachelor's degree, whereas one participant (7\%) did not specify the highest level of nursing education. Eleven (73\%) of the nurses worked in medical-surgical units, two nurses (13\%) worked in interventional areas (cardiac MRI and radiology), and two nurses (13\%) worked in PACUs.

The ABCDE score represented the assessment and management component. On the basis of the required elements for each scenario, the total ABCDE score could range from 1 to 36. Thirty-six indicated that all required elements for the scenario were addressed. The SBAR represented the reporting of clinical deterioration, and scores could range from 1 to 20. The total RAPIDS was the sum of the total ABCDE and total SBAR; possible scores ranged from 2 to 56 . The data were entered into an Excel spreadsheet and analyzed with Microsoft Excel 2010 (Microsoft Corp, Redmond, WA). The lowest and highest score achieved by any participant during baseline and after the intervention by use of the RAPIDS-Tool is shown in Table 1.

The mean score of each element of the assessment and management portion of the RAPIDS-Tool was calculated to assess baseline and post-intervention performance. Baseline performance generated a mean total score of 18.27, whereas the mean post-intervention score was 21.27. The mean total reporting scores were 11.33 and 13.33 for baseline and after the intervention, respectively. The total RAPIDS score increased from 29.60 at baseline to 34.60 after the intervention as depicted in Table 1.

Table 1. Simulation Score Ranges and RAPIDS Mean Scores

\begin{tabular}{llllll}
\hline \multirow{2}{*}{ Tool Component } & Variable & $\begin{array}{l}\text { Baseline } \\
\text { M (SD) }\end{array}$ & $\begin{array}{l}\text { Baseline } \\
\text { (Lowest/Highest) }\end{array}$ & $\begin{array}{l}\text { Post-Intervention } \\
\text { M (SD) }\end{array}$ & $\begin{array}{l}\text { Post-Intervention } \\
\text { (Lowest/Highest) }\end{array}$ \\
& Airway & $0.60(0.51)$ & $0 / 1$ & $0.07(0.26)$ & $0 / 1$ \\
ABCDE & Breathing & $4.60(1.18)$ & $2 / 6$ & $5.27(0.88)$ & $4 / 6$ \\
(Assessment and & Circulation & $5.67(1.59)$ & $3 / 8$ & $7.33(2.35)$ & $4 / 11$ \\
Management of & Disability & $0.73(88)$ & $0 / 3$ & $0.87(0.83)$ & $0 / 2$ \\
Clinical & Expose/Examine & $1.33(0.90)$ & $0 / 3$ & $1.60(0.83)$ & $0 / 3$ \\
Deterioration) & Global Rating & $5.33(1.05)$ & $3 / 7$ & $6.13(1.68)$ & $3 / 9$ \\
& Total ABCDE (*) & $18.27(3.49)$ & $11 / 25$ & $21.27(5.57)$ & $11 / 30$ \\
& Situation & $2.27(1.16)$ & $0 / 4$ & $2.80(1.37)$ & $0 / 4$ \\
SBAR & Background & $1.07(0.88)$ & $0 / 3$ & $1.33(0.90)$ & $0 / 3$ \\
(Reporting of & Assessment & $0.73(0.46)$ & $0 / 1$ & $0.80(0.41)$ & $0 / 1$ \\
Clinical & Recommendation & $0.60(0.51)$ & $0 / 1$ & $0.73(0.46)$ & $0 / 1$ \\
Deterioration) & Global Rating & $5.67(2.26)$ & $3 / 10$ & $6.73(2.09)$ & $3 / 9$ \\
& Total SBAR & $11.33(4.51)$ & $6 / 20$ & $13.33(4.05)$ & $6 / 18$ \\
& Total RAPIDS (*) & $29.60(5.84)$ & $22 / 41$ & $34.60(6.51)$ & $17 / 44$ \\
\hline
\end{tabular}

Note. The lowest and highest score achieved by any participant during baseline and after the intervention utilizing the RAPIDS-Tool and the mean RAPIDS scores before and after the intervention. Abbreviations: M, mean; RAPIDS, Rescuing a Patient in Deteriorating Situations Tool; SD, standard deviation.

*Significant difference at $p=.05$. 
Paired one-tailed $t$-tests were performed to compare the difference in mean baseline and post-intervention assessment and management scores as illustrated in Figure 1. There was a significant difference in mean baseline and post-intervention ABCDE scores $[t(14)=2.04, p=.03]$.

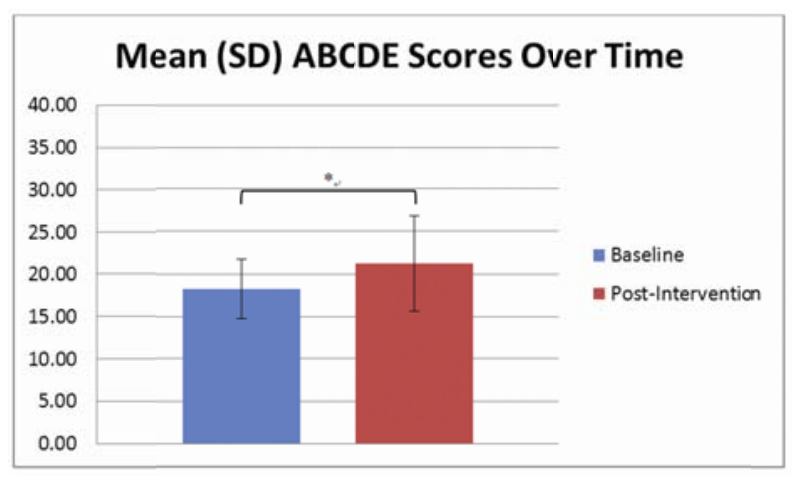

Figure 1. Mean ABCDE data comparing cumulative baseline and post-intervention performance (*significant difference at $p=.05)$

Paired $t$-tests were performed to determine the difference in mean SBAR scores between baseline and after the intervention. Again, calculations were based on one-tailed tests. There was no significant difference between mean baseline and post-intervention SBAR scores $[t(14)=1.58, p=.68]$ as shown in Figure 2.

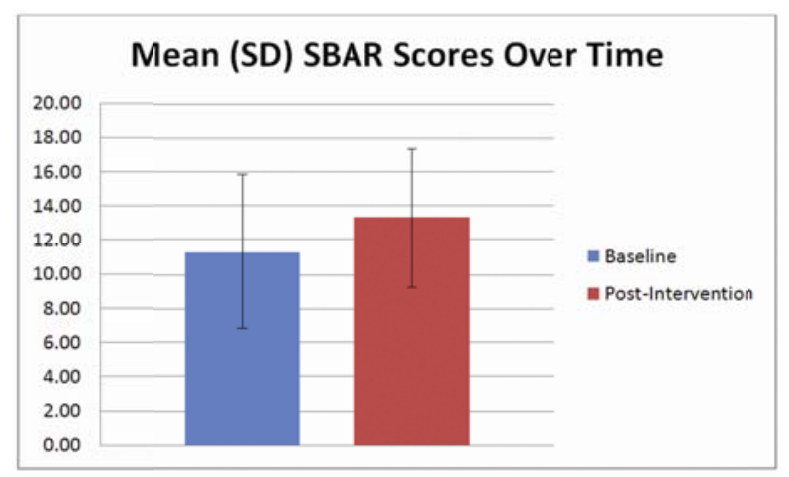

Figure 2. Mean SBAR data comparing cumulative baseline and post-intervention performance

Paired one-tailed $t$-tests were performed on the RAPIDS data as a whole. The $t$-tests evaluated the difference between the mean total baseline RAPIDS score and the mean total post-intervention RAPIDS score. As shown in Figure 3, there was significant difference $[t(14)=3.15, p=.004]$.

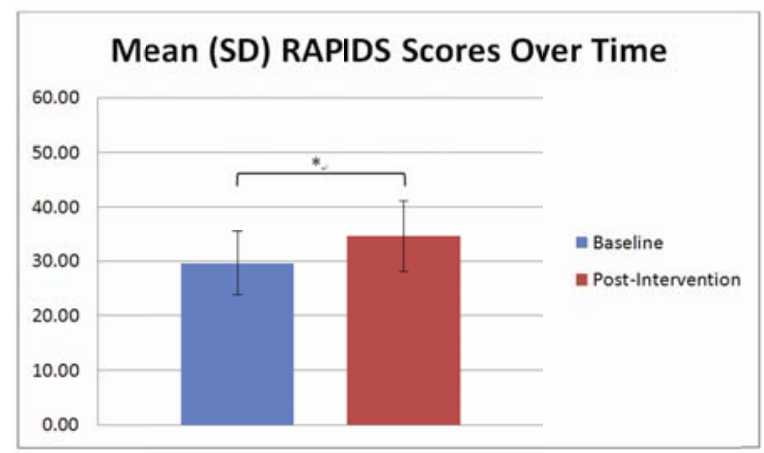

Figure 3. Mean total RAPIDS data comparing cumulative baseline and post-intervention performance (*significant at

$$
p=.05)
$$




\section{Discussion}

The results of this evidence-based practice project are consistent with the literature demonstrating that simulation-based education improves nursing knowledge of the recognition and management of clinical deterioration. All assessment variables increased after the intervention with the exception of the airway variable. A potential rationale for the lack of improvement in the airway variable is that the participants were more focused on executing newly presented information during the second scenario as opposed to this more instinctive variable. Participants self-reflected during the debriefing, which allowed for discussion on performance, rationales for actions, and opportunities for improvement.

The reporting variables (SBAR) did not reflect a statistically significant difference between the baseline and postintervention scores. However, there was a trend suggesting improvement. SBAR is the communication framework that has been promoted at the project facility for several years. Therefore, it is possible that familiarity with the SBAR components did not lend itself to the participants' appreciating new knowledge.

Although not part of the initial scope of the project, the correlation between simulation performance and years of nursing experience was evaluated. No correlation between simulation performance and years of experience was noted. Overall, the project was effective in increasing knowledge in the recognition of clinical deterioration.

A smaller than expected sample size was appreciated for several reasons, including the limited number of sessions offered. Simulator access was limited because it was shared with other departments. Also, room availability, time constraints, and participant cancellations led to decreased participation.

Project participation was voluntary and included nurses from medical-surgical units, procedural areas, and PACUs. An approach that may increase sample size would be to narrow recruitment efforts to one unit with the goal of having all nurses participate.

One simulation session was cancelled when the simulator became inoperable. Having access to a second simulator would decrease the possibility of having to cancel sessions owing to equipment malfunction.

Conducting both simulation scenarios on the same day limited the ability to assess long-term retention of the assessment, management, and reporting information garnered during the debriefing. Both scenarios were conducted on the same day because of the project's narrow timeline and concern that the participants may not return on a different day for a separate session. Also, the lack of long-term follow-up precluded evaluation of implementation in clinical practice.

\section{Conclusion}

A systematic approach to the assessment, management, and reporting of clinical deterioration with the use of mnemonics may enhance a nurse's performance and minimize omissions. The use of a validated tool provides objective data and aids in eliminating bias when evaluating clinical performance in a simulated environment. This evidence-based practice project demonstrated statistically significant improvement in the assessment, management, and overall post-intervention scores. These results are consistent with the literature indicating that simulation-based education may positively impact a nurse's knowledge of clinical deterioration.

\section{Acknowledgements}

To our colleague Theresa Pinn, RN, BS, we are appreciative of your support and assistance with the simulation sessions. To the volunteers that participated in this project, we thank and commend you for your interest in evidence-based practice. 


\section{References}

[1] Rattray JE, Lauder W, Ludwick R, Johnstone C, Zeller R, Winchell J, et al. Indicators of acute deterioration in adult patients nursed in acute wards: a factorial survey. J Clin Nurs. 2011; 20: 723-32. PMid:21320201

http://dx.doi.org/10.1111/j.1365-2702.2010.03567.x

[2] Endacott R, Scholes J, Buykx P, Cooper S, Kinsman L, McConnell-Henry T. Final-year nursing students' ability to assess, detect and act on clinical cues of deterioration in a simulated environment. J Adv Nurs. 2010; 66(12): 2722-31. PMid:20735506 http://dx.doi.org/10.1111/j.1365-2648.2010.05417.x

[3] McQuillan P, Pilkington S, Allan A, Taylor B, Short A, Morgan G, et al. Confidential inquiry into quality of care before admission to intensive care. BMJ. 1998; 316: 1853-8. http://dx.doi.org/10.1136/bmj.316.7148.1853

[4] Beaumont K. Deterioration in hospital patients: early signs and appropriate actions. Nurs Stand. 2008; 23(1): 43-8. PMid:18814476 http://dx.doi.org/10.7748/ns2008.09.23.1.43.c6653

[5] Steen C. Prevention of deterioration in acutely ill patients in hospital. Nurs Stand. 2010 Aug; 24(49): 49-57. http://dx.doi.org/10.7748/ns2010.08.24.49.49.c7935

[6] Cummings J, Krsek C, Vermock K, Matuszewski K. Intensive care unit telemedicine: Review and consensus recommendations. Am J Med Qual. 2007; 22: 239-50. http://dx.doi.org/10.1177/1062860607302777

[7] Chinn PL, Kramer MK. Integrated theory and knowledge development in nursing, 8th ed. St. Louis (MO): Elsevier Mosby; 2011.

[8] Clinical deterioration. In: Abate, FR, ed. Oxford pocket dictionary and thesaurus. New York (NY): Oxford University Press; 1997: 134-201.

[9] Liaw SY, Scherpbier A, Klainin-Yobas P, Rethans JJ. A review of educational strategies to improve nurses' roles in recognizing and responding to deteriorating patients. Int Nurs Rev. 2011; 58: 296-303. PMid:21848774 http://dx.doi.org/10.1111/j.1466-7657.2011.00915.x

[10] Buykx P, Kinsman L, Cooper S, McConnell-Henry T, Cant R, Endacott R, Scholes J. FIRST2ACT: Educating nurses to identify patient deterioration. A theory-based model for best practice simulation education. Nurs Educ Today. 2011; 31: 687-93. PMid:21481992 http://dx.doi.org/10.1016/j.nedt.2011.03.006

[11] Liaw S, Rethans J, Scherpbier A, Piyanee K. Rescuing a patient in deteriorating situations (RAPIDS): A simulation-based educational program on recognizing, responding and reporting of physiological signs of deterioration. Resuscitation. 2011; 82: 1224-1230. http://dx.doi.org/10.1016/j.resuscitation.2011.04.014

[12] Liaw S, Scherpbier A, Klainin-Yobas P, Rethans J. Rescuing a patient in deteriorating situations (RAPIDS): An evaluation tool for assessing simulation performance on clinical deterioration. Resuscitation. 2011; 82: 1434-9. http://dx.doi.org/10.1016/j.resuscitation.2011.04.014

[13] Mayville ML. Debriefing: The essential step in simulation. Newborn Infant Nurs Rev. 2011; 11(1): 35-9. http://dx.doi.org/10.1053/j.nainr.2010.12.012 